\title{
Les ateliers du chemin de fer de Tours et de sa
} région

Railway workshops in the region of Tours

Bernard Monteil et Christine Colombier

\section{OpenEdition}

\section{Journals}

Édition électronique

URL : https://journals.openedition.org/rhcf/1755

DOI : 10.4000/rhcf.1755

\section{Éditeur}

Rails \& histoire

\section{Édition imprimée}

Date de publication : 1 décembre 2003

Pagination : 73-99

ISBN : 0996-9403

ISSN : 0996-9403

\section{Référence électronique}

Bernard Monteil et Christine Colombier, «Les ateliers du chemin de fer de Tours et de sa région », Revue d'histoire des chemins de fer [En ligne], 28-29 | 2003, mis en ligne le 19 décembre 2014, consulté le 22 avril 2022. URL : http://journals.openedition.org/rhcf/1755; DOI : https://doi.org/10.4000/rhcf. 1755 


\section{Les ateliers du chemin de fer de Tours et de sa région}

\section{Les ateliers de Tours}

\section{La fusion des gares de Tours}

Le dualisme des gares de Tours créait de grosses difficultés d'exploitation. Ainsi, la ligne de Châteauroux mise en service par le réseau de l'État en 1880 et rétrocédée à la Compagnie du Paris-Orléans (PO) en 1883 aboutissait à la gare de l'État. Par contre, la ligne de Sargé ouverte en 1893 par l'État aboutissait à la gare d'Orléans. De plus, les deux gares n'étaient raccordées que par des plaques tournantes imposant des manœuvres longues et compliquées. Quant aux ateliers du PO ils étaient enclavés entre les voies des deux réseaux.

Cette situation aboutit en 1894 au dépôt d'un projet consistant en :

- la modification des raccordements aux abords de Tours;

- l'agrandissement de la gare de Saint-Pierre-des-Corps ;

- la fusion des gares de Tours-État et de Tours-PO et leur agrandissement.

De plus, à la demande de la ville de Tours qui offrait une subvention de 600000 F, la Compagnie d'Orléans accepta de reculer la façade de la gare de $70 \mathrm{~m}$ de façon à dégager l'accès à la rue de Bordeaux.

Le recul de la façade a diminué les emprises de la gare de $11000 \mathrm{~m}^{2}$. Cette nouvelle disposition entraîne des modifications dans l'avant-gare. C'est ainsi qu'un terrain d'une surface de 600000 ares doit être acquis dans la fourche constituées par les lignes de Saint-Pierredes-Corps et de Bordeaux pour construire le dépôt. Les emprises libérées par le dépôt permette à droite d'allonger les quais et les cours des marchandises, à gauche de dégager les ateliers ${ }^{1}$.

Le recul de la façade de la gare aura également pour conséquence le déplacement d'un grand bâtiment et d'un faisceau de voies, de l'atelier du petit entretien et des messageries. Les installations de l'atelier ont été déplacées sur des terrains libérés par le dépôt et par l’État.

1- Couvrat ; Sabouret, «La transformation des gares de Tours et Saint-Pierre-desCorps ", Revue générale des chemins de fer, septembre 1899, p. 137-151. 
Quant au petit entretien, il est réinstallé dans la gare voyageurs État et les messageries dans les nouveaux bâtiments de la gare voyageurs. La nouvelle gare, dont la façade est l'œuvre de l'architecte Victor Lalou, a été mise en service le 15 octobre $1898^{2}$.

\section{Les ateliers}

Après le réaménagement de la gare, la Compagnie du ParisOrléans envisage de décentraliser à Tours ses ateliers de Paris-Ivry. Dans cet esprit, l'ingénieur en chef du service Matériel et Traction demande en avril 1905 au chef d'arrondissement Voie et Travaux une étude sur l'extension des ateliers en vue de ce transfert. Un premier projet est présenté au ministre des Travaux publics en février 1907, il n'est pas retenu.

Ce n'est que le 8 mars 1910 que le ministre informe par lettre le préfet d'Indre-et-Loire des dispositions définitivement arrêtées. Elles portent sur l'agrandissement des ateliers qui « seront réservés à la réparation des machines, tenders et voitures à voyageurs $»^{3}$.

Néanmoins, «la compagnie renonce à concentrer à Tours, où elle ne dispose que de terrains insuffisants pour préserver l'avenir, tous les ateliers qu'elle est obligée de construire ou de prévoir tant à cause de l'extension des ateliers de Tours qu'à cause du transfert de ceux d'Ivry et de la nécessité de céder des terrains pour l'amélioration du service de l'Exploitation de la gare de Tours $»^{4}$.

De la description de ces futurs ateliers on retiendra : «Dans la bande est maintenue seraient conservés les bâtiments affectés aux ferreurs, aux bureaux et aux tours à roues, à la suite desquels on construirait des lavabos de 300 places[...] on conserverait le bâtiment du nouveau montage[...] on construirait trois vestiaires, l'un à 200 places, l'autre à 220 places $^{5}$. »

Sur la structure des bâtiments on apprendra que : « l'atelier d'ajustage en fer et en briques mesure $4350 \mathrm{~m}^{2}[. .$.$] L'atelier de forge aussi en$ fer et en briques occupe $1650 \mathrm{~m}^{2}$.»

Nous avons là une description certes assez sommaire mais qui permet d'imaginer l'importance et l'architecture de ces ateliers (fig.1 à 4). Ils seront réalisés conformément au projet.

\section{2- Ibid.}

3- Lettre du 8 mars 1910 du ministre des Travaux publics au préfet d'Indre-et-Loire, Archives départementales d'Indre-et-Loire.

4- Ibid.

5- Ibid. 


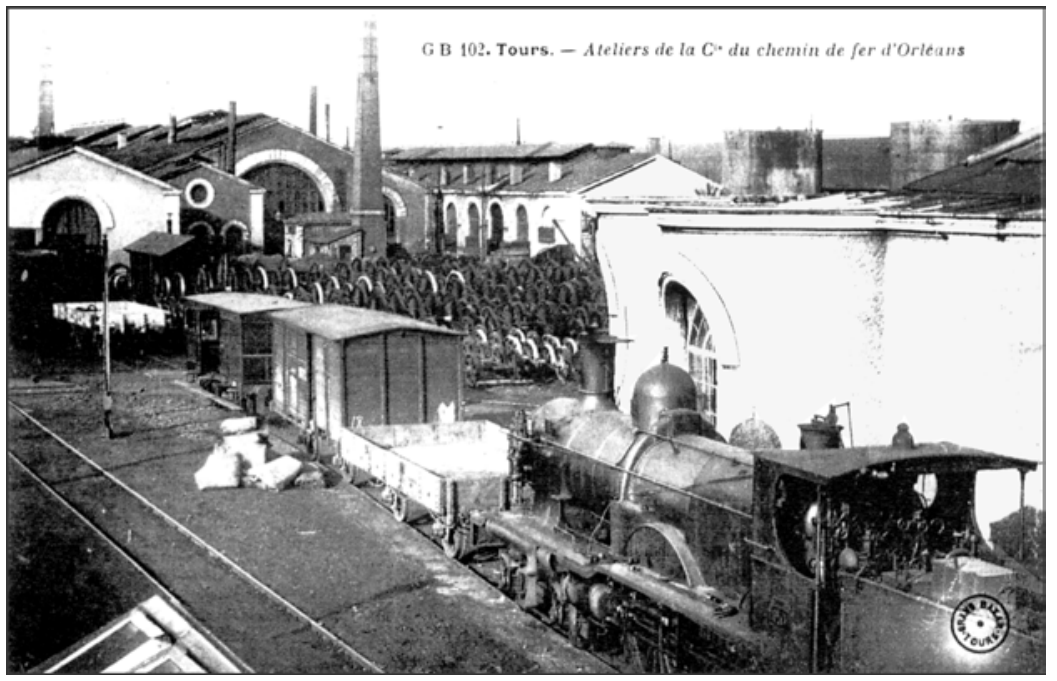

Figure 1. La rotonde du Tours-Nantes incorporée dans les ateliers PO vue depuis la passerelle pour piétons, vers 1900 . Cliché pris à la demande du PO et reproduit en carte postale. Coll. bibliothèque municipale de Saint-Pierre-des-Corps.

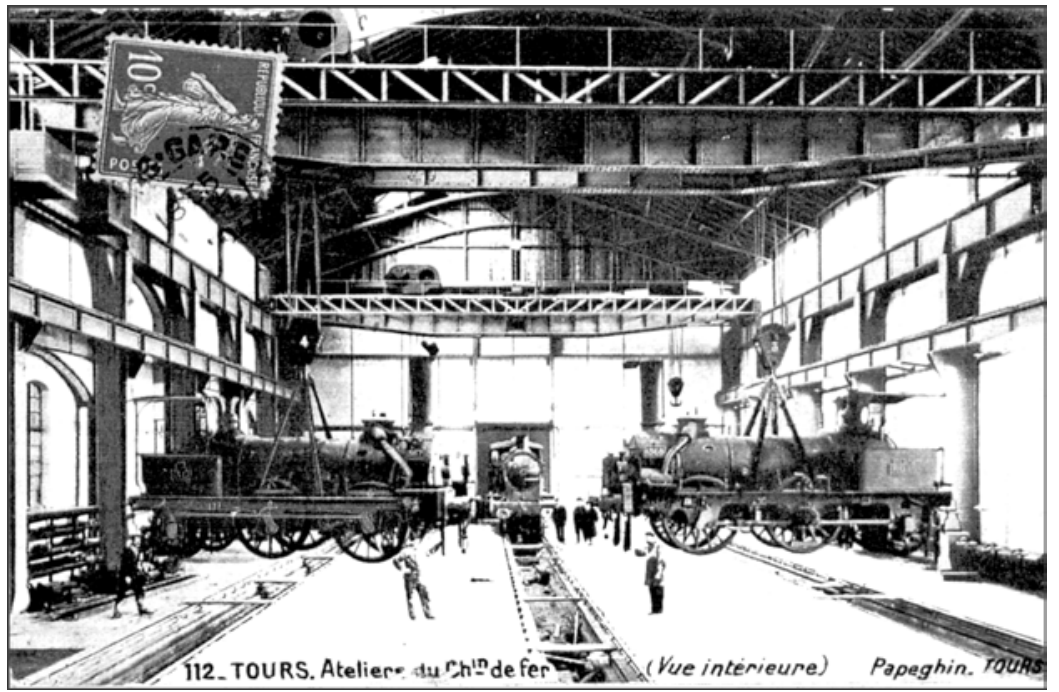

Figure 2. La grande halle de montage avec ses ponts roulants de $50 \mathrm{t}$, vers 1910. Cliché pris à la demande du PO et reproduit en carte postale. Cl. Papaghin, Tours. Coll. bibliothèque municipale de Saint-Pierre-des-Corps. 


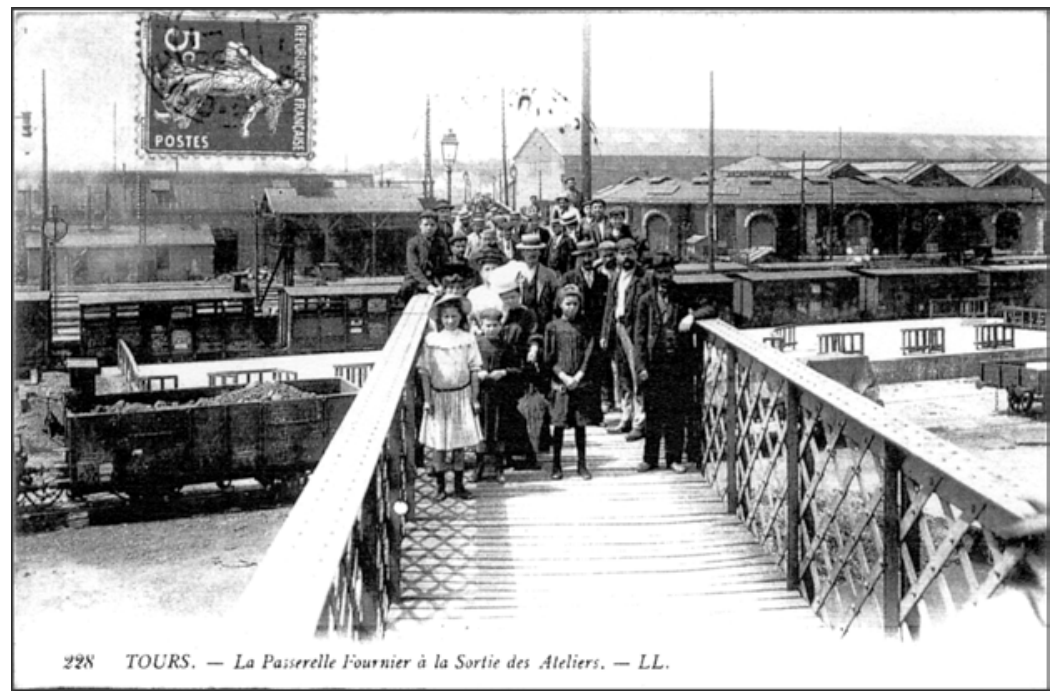

Figure 3. Passerelle "Fournier" pour piétons, vers 1910. La vue vers I'Ouest montre à droite, au premier plan, la rotonde de la Compagnie du Tours-Nantes, le plus ancien dépôt encore existant après 1898, construiten 1846 pour 16 locomotives. Derrière, le grand hall de montage (voir vue intérieure, fig. 2). Cliché pris à la demande du PO et reproduit en carte postale. Cl. LL, coll. J. Metz.

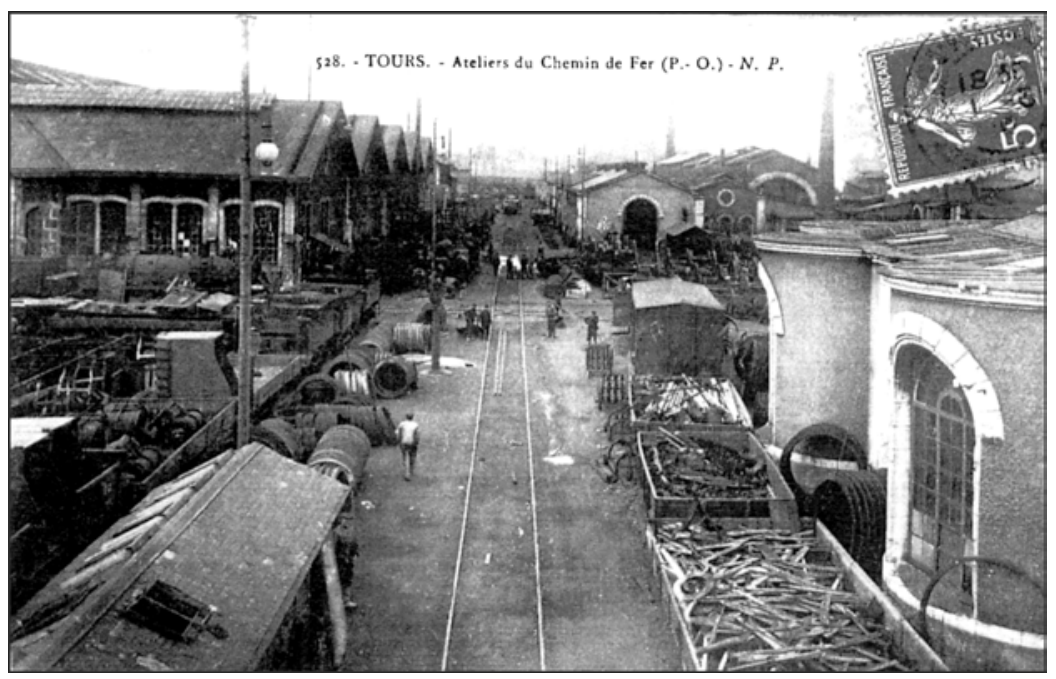

Figure 4. Au fond, les ateliers des locomotives, chaudronnerie et ateliers des roues et forges. Au premier plan à droite I'ancienne rotonde T.N., vers 1910. Cliché pris à la demande du PO et reproduit en carte postale. Cl. N.P., coll. J. Metz. 
Quant aux effectifs, il était prévu d'employer au moins 720 ouvriers, auxquels il faut ajouter les employés, les agents de maitrise et les cadres, soit un total de plus de 800 personnes.

Notons qu'en 1910 ont été ouverts à Saint-Pierre-des-Corps les ateliers d'entretien des wagons.

\section{La situation durant la Première Guerre mondiale}

Comme beaucoup d'ateliers et d'usines de toutes branches de l'industrie, les ateliers de Tours durent adapter leur production à la production militaire. On note également qu'en 1917 une compagnie de l'armée des États-Unis a été affectée à la réparation des locomotives.

\section{La vie dans les ateliers}

L'implantation et l'extension des ateliers ont entrainé l'urbanisation des quartiers sud de la ville. En contre partie, cette grande activité industrielle n'a pas été sans créer des nuisances. Nous citerons par exemple le bruit et la fumée des forges, les émanations dues au décapage des pièces et aux traitements thermiques. Dans les rues adjacentes, la circulation est intense, on croise beaucoup de cyclistes. Aux heures d'embauche et de débauche, c'est un véritable chassé-croisé. Les principales entrées des ateliers sont situées au 78, rue Blaise-Pascal, et rue du Sanitas. Elles sont gardées par des agents de surveillance en uniforme. Ils sont très physionomistes et ne laissent entrer que le personnel autorisé. Pour faire presser les retardataires, la sirène retentit à plusieurs reprises. À l'intérieur, le travail commence au même instant sur un coup de klaxon. Malgré une mécanisation avancée et des consignes et mesures de sécurité les conditions de travail sont dures ; l'effort physique nécessaire à la réalisation de certaines tâches reste important. Le bruit, la poussière, la chaleur ou le froid sont le lot quotidien de tous.

\section{Les méthodes de travail}

Soucieuse d'abaisser ses coûts de production, la Compagnie du Paris-Orléans instaure dans ses ateliers une politique de rationalisation du travail selon la méthode de Taylor. Outre la baisse des coûts de réparation et de maintenance, elle obtient également une production maximale de son parc de traction. Nous citerons, pour l'illustrer, l'exemple de la locomotive $n^{\circ} 4040^{6}$.

Entrée le 26 janvier 1931, elle est sortie douze jours plus tard apte à reprendre son service. Tous les organes sont visités et réparés, bon nombre sont remplacés. Le nombre de pièces ainsi examinées est

6- « Record », PO Illustré, n 15 (mai 1931), $3^{\text {e }}$ de couverture. 
estimé à plus de 1000 . Leur poids, leur dimensions sont variables. Un grand nombre sont confectionnées sur place pendant l'immobilisation de la machine en raison de l'absence de stock en magasin; dans le cas qui nous concerne, la chaudière nécessitant de gros travaux a simplement été échangée. À la révision de ce type de machine 10000 heures de travail sont allouées, dont 4000 pour la chaudière.

Avant 1921, l'immobilisation d'une machine pouvait atteindre 90 jours. À partir de cette année-là, le séjour fut réduit à 60 jours.

Les recherches poursuivies sur la rationalisation du travail dans les ateliers du Paris-Orléans ont abouti à une immobilisation moyenne de 24 jours à la fin des années 1930, réparation de la chaudière incluse.

Cela fait 12580 heures de travail en 1921, 8653 heures en 1930 (soit un gain de production de 31,22\%).

L'application de ces méthodes d'organisation du travail se concrétisa pleinement lors des travaux de transformation de locomotives entreprises à l'initiative d'André Chapelon, alors ingénieur chef aux bureaux d'études du matériel de la compagnie. Nous citerons pour exemple la reconstruction des douze Pacifics 2314500 en 2404700 (240-701 à 712). Après la sortie du prototype 240-701 (4701) le 16 août $1932^{7}$, la reconstruction des onze autres machines se réalisa en temps record de cinq mois (novembre 1933- avril 1934).

Il s'agissait en fait d'un véritable travail à la chaîne qui consistait en :

- la préparation en série des pièces neuves, cylindres HP (haute pression) et blocs du groupe BP (basse pression) par la fonderie ainsi que les entretoises, crosses, pistons, mécanisme distributeur, essieux coudés, foyers, viroles surchauffeurs par les forges?.

- Réparation des pièces conservées lors de la rentrée des machines aux ateliers.

- Montage des chaudières en six postes et des machines en cinq postes $^{10}$.

Le service de fabrication établissant :

- les demandes de matières premières.

7- L. Villain, Un Siècle de traction sur le réseau d'Orléans (1838-1940), réed., Paris, TardyLangelé/APRODEF.

8- Bernard Collardey ; André Rasserie, Les Locomotives à vapeur unifiées 241 P, 240 P, 150 P, Paris, Ed. La vie du rail et des Transports, 2001, 199 p.

9- Maurice Maillet, Les «Pacifics» PO-Midi et l'cuuve d'André Chapelon, Breil-sur-Roya, Ed. du Cabri, 1980.

10- Bernard Collardey; André Rasserie, op. cit. 
- Les procédés de fabrication des pièces neuves, l'inventaire des pièces conservées.

- Les phases de montage.

Les services de réparation, le magasin général s'assuraient de la réparation des pièces finies et fixaient les délais de séjours de chaque machine.

Le service du contrôle s'assurait de la qualité du travail. Le service technique veillait à la dépense et comparait les prix de revient avec les devis établis. Grâce à cette organisation, la durée de séjour d'une machine à l'atelier a pu être réduite à 35 jours et la cadence de sortie à trois jours ouvrables. Le premier semestre de 1934 vit la transformation de 42 machines : 11240 pour le PO, 31 Pacifics $^{11}$.

Outre les gains de productivité, la rationalisation du travail permettait une économie sur les installations. C'est ainsi que, disposant de dix emplacements pour réparer dix locomotives avec une immobilisation de 60 jours, avec les mêmes installations il était possible d'en traiter le double en 35 jours $^{12}$.

\section{La situation des années 1930-1940}

La mise en service en juillet 1933 de la traction électrique entre Paris et Tours ne modifia pas l'activité des ateliers. La fusion en 1934 des Compagnies du Paris-Orléans et du Midi amène l'apport des ateliers de Bordeaux.

La nationalisation des anciens réseaux intègre les ateliers de Tours à la SNCF région Sud-Ouest, arrondissement de Tours.

En 1939, les ateliers de Tours employaient 1700 salariés $^{13}$.

\section{La Seconde Guerre mondiale}

Lors de la déclaration de guerre, outre les révisions, les ateliers de Tours entreprenaient la construction et la transformation des locomotives 231 A-700 en 240 P.

Les bombardements aériens qui précédèrent l'invasion allemande de juin 1940 n'atteignirent que les parties nord de la ville. Les ateliers situés au Sud ne furent pas endommagés. De ce fait, il purent reprendre rapidement leurs activités.

\section{1- Ibid.}

12- « Record», art. cit., mai 1931.

13- René Parès ; Lozé, « La reconstruction ferroviaire de Tours-Saint-Pierre-desCorps », RGCF, mai 1948, p. 150-160. 
En février 1943, ils subiront une première attaque aérienne. Venant par l'ouest, une escadrille de Mosquitos lâche ses bombes cinq minutes avant l'heure de sortie des ateliers. Aucune n'explosera sur le site mais le quartier subit d'importants dégâts, des morts sont à déplorer. Puis après un calme relatif ponctué par de nombreuses alertes, le 11 avril 1944 un bombardement neutralisa les installations ferroviaires de Saint-Pierredes-Corps. Certains pensaient que les objectifs étaient atteints et que la gare et les ateliers de Tours seraient épargnés. Il n’en fut pas ainsi car, dans la nuit du 19 au 20 mai 1944, une puissante attaque aérienne détruisit l'avant-gare en s'attaquant plus particulièrement aux ateliers, tuant 137 personnes.

\section{La Libération}

À la Libération, l'ensemble des installations ferroviaires de ToursSaint-Pierre-des-Corps était pratiquement inutilisable. De plus, elles étaient entièrement isolées du reste du pays du fait de la destruction des ouvrages d'art. En gare de Tours, seuls le bâtiment voyageurs et quelques bâtiments " grande vitesse » étaient normalement utilisables ; quant aux ateliers, les bâtiments et l'outillage avaient énormément souffert. L'atelier de chaudronnerie était entièrement détruit. Toutefois, l'atelier de montage, grâce à la robustesse de sa structure, était relativement épargné. De ce fait, les ponts roulants de 25 et 50 t l'ont été également. Ainsi, vu les besoins pressants de matériel, les ateliers reprennent une activité partielle, dans les locaux subsistants.

\section{La reconstruction et l'avenir}

Dès la Libération, la décision était prise après une réduction progressive de leur activité, les ateliers de Tours doivent être définitivement fermés. Début 1947, ils employaient encore 400 agents. Pour l'avenir il était prévu qu'un atelier de mécanique générale pour les pièces produites par la fonderie de fonte et de bronze de Saint-Pierre-des-Corps serait édifié sur le même site. De son côté, la ville de Tours souhaitait reprendre les terrains des emprises ferroviaires afin de les urbaniser. Dans cet esprit fut projetée la création d'une gare Tours-Saint-Pierredes-Corps. Elle se serait située sur la ligne de Nantes, à la limite des deux communes. Ces projets n'eurent pas de suite ${ }^{14}$.

Après de longues et laborieuses discussions entre, d'une part, le ministre de la Reconstruction et de l'Urbanisme, la ville de Tours et la SNCF d'autre part, un accord intervient le 19 décembre 1950. Il stipule : «Après les destructions de 1944 la SNCF sur la suggestion des 
urbanistes a envisagé des remaniements importants des installations ferroviaires [...] Les terrains de la gare de Tours appartenant à la SNCF devront être remis au MRU ${ }^{15}$ " pour la création d'artères nouvelles et la construction d'immeubles d'habitation ${ }^{16}$.

C'est sur ces bases que les deux dépôts État et Orléans ainsi que ceux des ateliers et voies de dessertes sont vendus : l'acte de vente est signé le 26 décembre $1956^{17}$.

Aujourd'hui, un quartier neuf, une université ont été construits sur ces emplacements. Ils ne subsiste aucun vestige des ateliers.

Une association composée d'anciens cheminots des ateliers et d'amis du rail s'est constituée pour perpétuer le souvenir de cet important complexe industriel et ferroviaire.

\section{Les ateliers de réparation du matériel ferroviaire de Saint-Pierre- des-Corps}

Cet atelier a une histoire peu commune puisque son personnel a appartenu tout d'abord à la Compagnie du Paris-Orléans (PO) jusqu'en 1920, puis au secteur industriel privé jusqu'en 1982, et a été intégré

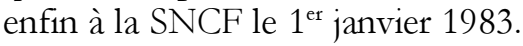

\section{L'époque du Paris-Orléans}

Nous tenons ici à relater les faits principaux de l'histoire sociale de cet atelier. Une histoire jalonnée, depuis des décennies, par la combativité des travailleurs qui s'y sont succédé.

Sa construction remonte à 1910, sous la direction de la Compagnie de chemin de fer du Paris-Orléans (PO). À cette époque, la réparation des wagons et la maintenance des locomotives sont effectuées dans l'emprise du réseau PO, derrière la gare de Tours. La construction des nouveaux ateliers de Saint-Pierre-des-Corps répond à un besoin pour l'entretien du matériel roulant de la compagnie. C'est un gros chantier qui durera plusieurs années. Une partie importante des matériaux (briques, sable, ciment) sont transportés par voie d'eau et débarqués sur le bord du canal sur un quai aménagé à proximité du pont de chemin de fer puis amenés à pied d'œuvre par un Decauville.

15- Ministère de la Reconstruction et de l'Urbanisme. 16- D’après Maurice Gaucher.

17- D’après Maurice Gaucher. 
Le 31 août 1914, les chemins de fer passent sous l'autorité militaire et ce, jusqu'au 2 février 1919. Pendant la guerre, les ateliers PO sont utilisés à des fins militaires, dont la réparation des vêtements de l'armée américaine, avec une main-d'œuvre féminine nombreuse. La réparation des moteurs d'avions ainsi que la fabrication de canons (selon les brevets du colonel d'artillerie Rimailho) ont lieu dans de grands hangars en bois montés par les Américains (fig. 5).

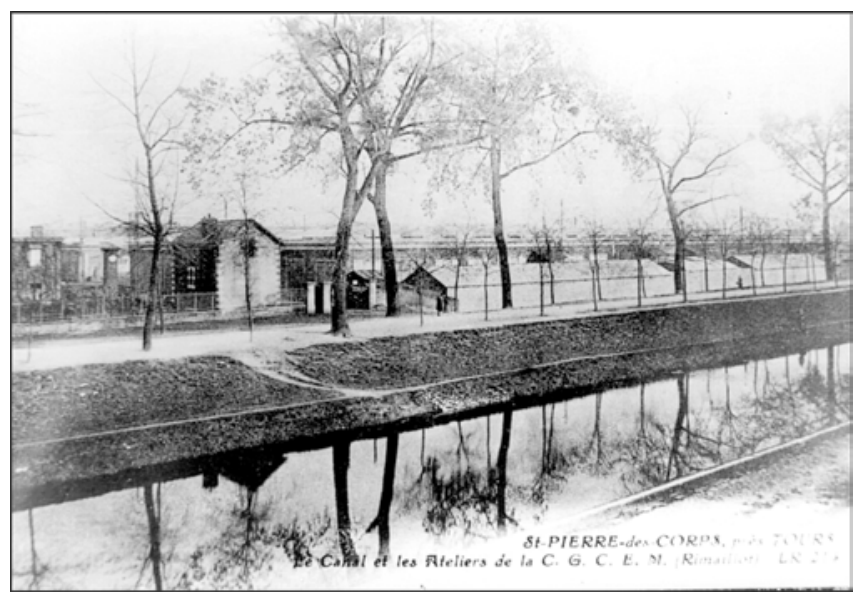

Figure 5. Tours-Saint-Pierre. Cité "Rimaillot" au début du siècle. Coll. bibliothèque municipale de Saint-Pierre-des-Corps.

Les cheminots tourangeaux qui, dans un premier temps, sont influencés par les partisans de l'Union sacrée, reprennent rapidement l'action pour de meilleures conditions de vie. En 1917, l'agitation a pour but d'obtenir des indemnités de cherté de vie et la suppression du travail à la tâche. La forte syndicalisation des cheminots explique leur combativité : 4550 agents sont syndiqués sur un effectif de 5350 et la proportion de cheminots syndiqués atteint $88 \%$ dans les ateliers.

Après la fin de la guerre, les ateliers $\mathrm{PO}$ retrouvent peu à peu leur vocation première de réparation du matériel ferroviaire.

Chez l'ensemble des cheminots, cette période d'après-guerre est marquée par une intense activité syndicale et politique. Après avoir participé aux grèves revendicatives de janvier et février 1920, le syndicat des cheminots tourangeaux s'associe à la grève de mai. Elle est très forte localement, en raison de l'importance des effectifs des cheminots (8 596), et en particulier chez les ouvriers des ateliers où la participation à l'action gréviste varie de 60 à $75 \%$. 
Cette grève, par son ampleur et la répression qui s'en suivit, marqua douloureusement l'histoire sociale du pays : plus de 2000 révocations et 4000 arrestations dont celles de Gaston Monmousseau et Pierre Sémard, responsables syndicaux et politiques. Pour le seul centre de Tours-Saint-Pierre-des-Corps, il y eut 2330 révocations et la fermeture des ateliers. Les grévistes révoqués ne trouvèrent plus de travail car ils figuraient sur des listes d'ouvriers « interdits d'embauche ».

C'est pour briser la grève des cheminots des ateliers d'entretien de Saint-Pierre-des-Corps que la direction du PO licencie tout le personnel et concède la gestion des ateliers à la CGCEM (Compagnie générale de construction et d'entretien du matériel) pour une durée de quinze ans.

Celle-ci place à sa tête comme nouveau directeur un ancien officier de l'armée française : le colonel Rimailho qui s'était illustré par l'invention d'un canon de 155 à cadence de tir rapide puis, dans le domaine économique, par la publication d'ouvrages sur des méthodes de travail au rendement inspiré du taylorisme.

Sa première tâche est de reconstituer l'effectif, nécessaire à l'exploitation des ateliers. Il est alors composé en partie de cheminots mutés venants d'autres ateliers d'entretien de la Compagnie PO, de 700 cheminots révoqués pour « fait de grève » à l'exception des militants syndicalistes, ainsi que de nouveaux embauchés originaires de l'agglomération tourangelle.

Fin 1922, l'entreprise emploie une majorité d'anciens ouvriers du PO, 900 au total. A cette date, la lutte pour la réintégration commence. Les révoqués qui utilisent le sigle de la nouvelle société CGCEM pour se dénommer : "Cheminots grévistes congédiés en mai ", s'organisent rapidement en constituant un organisme de défense intitulé «Section des révoqués de Tours », dirigé par Bellœuvre. Pierre Sémard tient un meeting à Tours le 26 octobre 1923, réclamant l'amnistie. Le 2 novembre, réunion des cheminots révoqués qui revendiquent leurs droits; le 14 décembre, ils s'adressent au Bloc des gauches. Un accord intervient entre le ministre des Travaux publics et les compagnies ferroviaires en octobre 1924 pour la réintégration des révoqués. Mais les mesures de réintégration sont lentes et partielles. Les militants syndicaux, les ouvriers de la CGCEM et les cheminots ayant dépassé l'âge de la retraite sont exclus.

Jacques Valdour ${ }^{18}$, qui s'était fait embaucher comme aide ferreur aux ateliers de la CGCEM en juillet 1924, pour « mieux comprendre la

18- Publie en 1929 un ouvrage intitulé : La Menace rouge : Ouvriers d'après-guerre en Touraine, observations vécues. 
condition et la psychologie ouvrière ", décrit à plusieurs reprises la situation des agents révoqués en 1920. Bien que partisans et donc réducteurs, ses propos ne sont pas dénués d'intérêt.

J'entre aux ateliers comme ferreur ; à raison de 2 francs l'heure et 0,10 franc d'indemnité de vie chère. La durée quotidienne du travail est de 9 heures. La semaine anglaise n'est pas observée [...] Dans le hall de la Chaudronnerie, travaillent 200 ouvriers : forgerons, perceurs, soudeurs, ferreurs, tôliers... Mon équipe compte une douzaine d'hommes, dont un Bordelais et deux Périgourdins. C'est à l'un de ces derniers que je suis adjoint. "Vous n'apparteniez pas à la Compagnie autrefois ?", me demande-t-il presque aussitôt, faisant allusion aux révocations qui frappèrent un grand nombres des cheminots à la suite de la tentative de grève révolutionnaire du $1^{\text {er }}$ mai 1920 ; lui-même révoqué, avait dû quitter Périgueux, où il travaillait aux ateliers de la Compagnie d'Orléans, pour entrer aux ateliers de la filière indépendante où il perdait tout les avantages que lui accordait le statut de cheminot : gratifications, parcours gratuits, retraite assurée [...] Sur les 2500 ouvriers (il ne se trouve parmi eux qu'un petit nombre d'étrangers, 300 à peine, Italiens, Espagnols, Tchécoslovaques, Polonais, Russes, Grecs, Arméniens. Parmi les Français on trouve beaucoup de Bretons) de la filiale, 1500 sont des révoqués de 1920 [...] L'amnistie est votée, me dit un jour, de lui-même, Marcel de Périgueux, pendant que j'arrache à de vieux tuyaux utilisables leur enveloppe de toile et d'amiante ;" - Je vais attendre pour voir si la Compagnie accorde la réintégration; alors je retournerai au pays ; je serai aussi bien payé qu'ici et la vie y est moins chère." Je suis étonné du calme, de l'indifférence même avec laquelle il me parle de l'amnistie et de la réintégration. Pour les enfants, on apprend que, chez eux, en famille, beaucoup de révoqués s'en préoccupent. Mais entre eux, ils est extrêmement rare qu'ils y fassent allusion. Faisant route, un matin, jusqu'à l'atelier, avec un Périgourdin, pensionnaire à mon auberge et ferreur dans mon équipe : "- Eh bien! lui dis-je, et cette réintégration? Oh ! répond-il placidement, elle viendra par lettres individuelles ; chacun de nous, l'un après l'autre, on recevra avis, la Compagnie examinera chaque cas en particulier." Arrivés à l'atelier, nous attendons à nos établis le signal de la reprise du travail. Un Tourangeau dit ironiquement: "- Le dernier bénéficiaire de la réintégration l'obtiendra en 1950 au plus tard." Un autre Tourangeau répond tranquillement : "- Puisqu'il y a une loi, il faudra bien qu'elle soit appliquée." Mais lorsque le travail est commencé, près d'un étau où trois compagnons sont groupés, l'un deux, un grand gaillard, originaire d'une province du Centre, gesticule furieusement et crie : "-C'est pas dans dix ans qu'il nous faut la réintégration, c'est tout de suite ! Ou bien alors il nous 
faut la tête du patron !" C’est la seule remarque un peu passionnée que $j$ 'ai pu surprendre. Une autre fois, sur un ton d'impatience, un menuisier périgourdin, d'une vingtaine d'années, s'écria : "-Vingtcinq mille ont été révoqués en 1920. C’est dégoûtant !" ; un samedi matin le chef d'équipe me dit: "- Vous ne travailliez pas au chemin de fer avant de venir ici ? Non, jamais. Moi, si. Ils m'ont foutu à la porte. - En 1920 ? - Oui." Il me dit cela placidement, d'un air de complète indifférence. "- Bah! Répliquai-je, vous serez réintégré ! - Oh! répond-il du même ton lointain. Je ne crois pas. - Et pourquoi donc ? - Oh! Vous savez, la loi, quand on la regarde de près, entre les lignes, elle ne dit pas grand chose, à moins qu'elle ne dise le contraire de ce qu'elle a l'air d'accorder. J'ai pas confiance... Peuh! On n'est pas malheureux ici. Mais c'est tout de même pas la même chose que le chemin de fer... Oui, la retraite! Et puis les congés payés, les permis de circulation..."

\section{De l'industrie privée à la SNCF}

Un second accord signé en 1933 permettra la reprise de nombreux révoqués. Mais certains en seront encore exclus (ex-mineurs, apprentis, militants). On réintègre donc une partie des ouvriers, en leur octroyant les 42 et $48 \mathrm{~h}$ de travail et, pour les autres, 32 et même $28 \mathrm{~h}$.

La CGCEM exploite les ateliers de réparation de Saint-Pierredes-Corps jusqu'en 1931, date à laquelle lui succède la CIMT (Compagnie industrielle de matériel de transport).

La convention du 31 août 1937, stipulant que les compagnies privées de chemins de fer renonçaient, à compter du $1^{\text {er }}$ janvier 1938 et pour 45 ans, au droit d'exploitation les concessions, oblige celles-ci à leur unification au sein de la SNCF, elle-même créée par décret-loi de la même date.

Ainsi, le $1^{\text {er }}$ janvier 1937, les ateliers de réparation de Saint-Pierredes-Corps deviennent la propriété de la SNCF, comme tous les biens des compagnies privées des chemins de fer. Une seule exception demeure pourtant : le personnel de l'atelier de Saint-Pierre-des-Corps reste attaché à la société privée CIMT et se voit privé du statut de cheminot.

En septembre 1961, à l'occasion d'un renouvellement des marchés SNCF d'entretien du matériel remorqué confié au secteur privé, la Société Cadoux devient le nouveau locataire des bâtiments et des machines.

Début 1977, suite à une chute brutale de $15 \%$ du programme de réparations attribué par la SNCF à l'atelier de Saint-Pierre-des-Corps, les difficultés d'emploi surgissent, entrainant diminution d'horaire et chômage partiel. La direction SNCF ne cache pas son intention de continuer à réduire progressivement chaque année le programme confié à l'atelier de Saint-Pierre-des-Corps, avec la perspective d'aboutir à une extinction totale de son activité. 
Placé devant une telle situation où 860 emplois se trouvent menacés à terme, l'idée de l'intégration du personnel à la SNCF fait son chemin dans les esprits. Rapidement, elle s'avère la seule solution pour les emplois et le maintien de la réparation ferroviaire à Saint-Pierre-des-Corps.

Le 17 mars 1977, le syndicat CGT organise une consultation à bulletins secrets au cours de laquelle $96 \%$ du personnel se prononce pour son intégration à la SNCF.

Partant de cette décision, pendant six années, les luttes du personnel, impulsées par le syndicat CGT des cheminots - qui avait inscrit ce point important dans son cahier revendicatif - n’ont pas cessé de se développer pour la réintégration à la SNCF et s'opposer à tout licenciement (multiples actions et démarches en avril 1977, juillet 1981 ; le 5 mai 1979 : «Portes ouvertes »).

Devant une telle détermination des salariés dans leurs actions pour aboutir, le 6 mai 1982 le ministre des Transports, Charles Fiterman, fait savoir qu'il « vient de prendre la déscision d'apporter une conclusion heureuse à la lutte menée depuis de monbreuses années par les travailleurs des ateliers de Saint-Pierre-des-Corps, en demandant à la direction de la SNCF d'assurer elle-même l'exploitation de cet établissement et de mettre au point, en liaison avec les représentants du personnel, les modalités pratiques de sa réintégration ».

Le 4 juin 1982, en apprenant la décision du ministre des Transports de réintégrer à la SNCF tout le personnel de l'atelier de Saint-Pierre-des-Corps, Raoul Gerbault, gréviste de 1920 qui n’a jamais été réintégré, ne cache pas son émotion en déclarant : "Après 62 années de sanction disciplinaire, c'est notre réabilitation à tous que représente cette réintégration du ministre. »

C'est le lundi 3 janvier 1983 que devient enfin effective l'intégration au sein de la SNCF des 636 cheminots de l'atelier de Saint-Pierre-des-Corps, venant ainsi s'ajouter aux 60 agents de la mission de contrôle qui travaillent dans l'établissement.

Mais la direction du Matériel SNCF n’a pas renoncé, à terme, à la fermeture de l'atelier de réparation de Saint-Pierre-des-Corps et des magasins d'approvisionnement qui en dépendent (le magasin général des matières). Dans cet optique, de 1983 à 1992, elle ne cesse de réduire les effectifs et la charge de travail :

- $1^{\text {er }}$ janvier 1985 : rattachement à l'atelier de l'Entretien SNCF de Tours-Saint-Pierre-des-Corps (210 agents).

- $1^{\text {er }}$ décembre 1987 : rattachement du Magasin général (260 agents), les trois établissements n'en formant alors qu'un seul : l'atelier du Matériel (ATM). 
Le 28 septembre 1990, la direction de l'établissement informe les organisations syndicales du changement total d'orientation pris par la direction du Matériel SNCF concernant l'atelier de Saint-Pierre-desCorps, celui-ci devient atelier « concourant» de Vitry avec la décision de l'extinction totale des travaux sur « voitures classiques » remorquées, prévue pour la fin de l'année 1993.

À compter de cette date, l'atelier de Saint-Pierre-des-Corps ne devait plus traiter que du matériel moteur autotracté moderne à deux niveaux. Une mutation totale qui implique cependant de très importants travaux d'aménagement et de modernisation des ateliers.

Le $1^{\text {er }}$ janvier 1993, il devien EIMM : Établissement industriel de maintenance du matériel.

Durant dix ans, les cheminots des ateliers de Saint-Pierre-desCorps luttent pour s'opposer à la fermeture de leur établissement et au départ de certains travaux vers le secteur privé, tel le grenaillage de coques de sièges de VB2N. Mais cet établissement ne comptait plus au total, au $1^{\text {er }}$ janvier 1993, que 804 agents sur un effectif global de 1206 personnes en 1983, soit plus de 400 suppressions d'emplois en dix ans! Fin 1993, les actions menées par les cheminots portent leurs fruits. L'EIMM de Saint-Pierre-des-Corps est choisi par la direction de l'entreprise pour devenir l'atelier directeur de l'entretien des matériels automoteurs de la SNCF.

Au-delà de l'entretien des matériels destinés aux services régionaux, la charge de travail de l'EIMM s'oriente donc massivement vers le matériel circulant en Île-de-France.

\section{La fermeture des ateliers de grandes révisions de Vitry}

En juin 1993, la direction de la SNCF présenta au Comité central d'entreprise un projet visant à fermer la partie " grandes révisions » des ateliers de Vitry. Cette fermeture devait s'étaler dans le temps jusqu'à l'horizon 2000 avec un transfert progressif des activités sur le site de Saint-Pierre-des-Corps. Ce projet résulte d'un plan national, qui consiste à chercher un équilibre entre les établissements provinciaux et parisiens. Il tient compte de la charge de révision liée au renouvellement du matériel. Ce transfert concerne 540 emplois sur 700 .

Cette décision est immédiatement contestée par le comité régional d'établissement de Paris-Rive-Gauche (CRE). Il lui oppose une étude réalisée à sa demande par un cabinet spécialisé. Elle précise que : "l'objectif principal est un gain de productivité dans le cadre d'une politique de "maintenance conditionnelle" privilégiant la révision à l'organe et au kilomètre parcouru plutôt qu'une révision globale du 
matériel en fonction du temps, critère qui fondait jusqu'alors la démarche de la SNCF. Un matériel moderne, moins de maintenance, tout cela expliquerait la restructuration des établissements directeurs, avec la fermeture définitive des ateliers de La Folie à Nanterre. Elle cite également, à l'appui de ses affirmations, une série d'incidents survenus sur le matériel moderne. Enfin, elle déplore que la fermeture d'une partie du site de Vitry risque de priver la région parisienne d'un atelier de proximité. Pour conclure, l'étude propose la transformation de Vitry en centre de maintenance et de développement du matériel automoteur pour Paris-île-de-France.

Dans sa réponse, la direction de la SNCF précise que sa décision ne concerne que l'atelier des grandes révisions, qui sont effectuées tous les huit à dix ans. L'entretien resterait à Vitry, sur le site des Ardoines (cela représente 160 emplois et 23 hectares sur les 33 qu'elle exploite), que la sécurité, la fiabilité et la régularité ne seraient pas affectées par le choix de Tours.

Le transfert à Tours est aussi un choix social. La SNCF évoque pour se justifier l'impasse dans laquelle se trouvent les ateliers de SaintPierre-des-Corps avec l'arrêt des révisions de la génération des voitures précédant le « Corail » et l'avenir de ses 800 emplois.

En région parisienne l'ensemble des services du Matériel représente 6900 emplois, la fermeture de l'atelier de grandes révisions de Vitry créerait moins de difficultés de reclassement. La SNCF s'appuie pour cela sur les demandes de mutations en province. Elle informe que près de 200 emplois sont réservés à Tours, Limoges, Périgueux et Bordeaux avec les mesures financières d'accompagnement. Quand aux agents mutés dans d'autres établissements de la région parisienne, elle précise que ce sont les emplois et non les personnes qui sont délocalisées.

En conclusion, la direction de la SNCF rejette l'étude proposée par le CRE et maintient sa décision de fermeture. De son côté, la ville de Vitry rejette également la délocalisation de ses ateliers. Elle y voit la perte d'une partie de son potentiel industriel et humain. Pour tenter de la rassurer, la direction de la SNCF lui précise son intention d'agrandir le banc d'essai des machines qui s'y trouve par la construction d'un nouveau bâtiment et l'arrivée de 80 agents. Elle tient à lui préciser que cela lui maintiendrait la même taxe professionnelle.

\section{L'EIMM de Saint-Pierre-des-Corps}

Avec le transfert des activités « grandes révisions » de Vitry, les Ateliers du matériel de Saint-Pierre-des-Corps deviennent, on l'a dit, «Établissements industriels de maintenance du matériel de Saint-Pierre- 
des-Corps ", le $1^{\text {er }}$ janvier 1993. À ce titre, ils se voient chargés de la maintenance du matériel automoteur francilien et TER. Cette mutation entraine une restructuration profonde des ateliers. Le montant des investissements est estimé à environ $200 \mathrm{MF}$.

Après les travaux, les établissements disposent des installations suivantes :

- Superficie du chantier central : 15 ha.

- Superficie couverte : 5 ha.

- Nombre de bâtiments : 9.

- Faisceau : 10 voies de $350 \mathrm{~m}$, y compris deux d'essai.

- Deux chariots transbordeurs : 70 t pour véhicules de $25 \mathrm{~m}$, $80 \mathrm{t}$ pour véhicules de $27 \mathrm{~m}$.

- Nombres de postes de travaux sur caisses : 68.

- Installation de grenaille: une grenailleuse à turbine. Une grenailleuse pilotée par automates semi-automatique, gain de temps : $30 \%$, se qui représente une importante amélioration des conditions de travail.

- Installations de peinturage : une cabine d'enduisage de $29 \mathrm{~m}$, une cabine de peinture de $29 \mathrm{~m}$, un tunnel de séchage de $29 \mathrm{~m}$.

- Chantiers particuliers : complexe de nettoyage (caisse/bogie). Chambre RA. Chambre de collage élastomère. Installation de détartrage. Cabines de peinture bogie, moteurs de traction, pièces de diverses origines. Four à lit de sable. Installation de poudrage. l'effectif).

- Effectifs : 980 ; hommes : 905 ; femmes : 75 (soit 7,65\% de

Par collège :

\begin{tabular}{|l|c|c|r|}
\hline & Cadres & Maitrise & Exécut \\
\hline Hommes & 47 & 234 & 624 \\
\hline Femmes & 6 & 16 & 53 \\
\hline
\end{tabular}

Par service:

\begin{tabular}{|l|c|}
\hline Services & Nombre d'agents \\
\hline $\mathrm{P}$ & 650 \\
\hline $\mathrm{A}$ & 150 \\
\hline $\mathrm{T}$ & 85 \\
\hline GE & 30 \\
\hline Transverses & 65 \\
\hline
\end{tabular}

Source SNCF.

Tableau 1. Répartition par collèges et par service de l'effectif de I'EIMM de Saint-Pierre-des-Corps en 2001. 
Certains postes ont été aménagés pour être occupés par des agents handicapés et plus particulièrement pour des malentendants.

L'établissement de Saint-Pierre-des-Corps renoue également avec la tradition un peu oubliée : l'apprentissage. Actuellement, il assure en partenariat avec un établissement scolaire la formation pratique d'apprentis aspirant aux CAP, BEP. Ils auront la possibilité à l'issue de leurs études d'être embauchés.

\section{Les réalisations}

Au 31 décembre 2001 l'EIMM de Saint-Pierre-des-Corps est atelier directeur de 1023 automotrices se répartissant ainsi :

- Île-de-France : 697

- TER : 304

- Luxembourg (CFL) : 22.

\begin{tabular}{|l|c|c|r}
\hline \multicolumn{1}{|c|}{ Série } & Nombre & Affectation & Affectal \\
\hline$Z 5100$ & $\mathbf{2 0 0 1}$ & I.D.F. & T.EF \\
\hline$Z 5300$ & 141 & 113 & 28 \\
\hline$Z 5600$ & 52 & 52 & \\
\hline$Z 6100$ & 75 & 75 & \\
\hline$Z 6300$ & 33 & 12 & 21 \\
\hline$Z 6400$ & 75 & 75 & \\
\hline$Z 8100$ & 51 & 51 & \\
\hline$Z 8800$ & 58 & 58 & \\
\hline$Z 20500$ & 194 & 194 & \\
\hline$Z 20900$ & 17 & 17 & \\
\hline$Z 22500$ & 53 & 53 & \\
\hline$Z 920500$ & 6 & & 6 \\
\hline$Z 7300$ & 75 & & 15 \\
\hline$Z 7500$ & 15 & & 20 \\
\hline$Z 9500$ & 20 & & 36 \\
\hline$Z 9600$ & 36 & & \\
\hline$Z 11500$ & 22 & & \\
\hline$Z 23500$ & 80 & & \\
\hline$L U X$ & 22 & & \\
\hline
\end{tabular}

Source : SNCF

Tableau 2. Les automotrices affectées à l'EIMM de Saint-Pierre-desCorps en 2001. 
Parmi toutes ces réalisations retenons principalement :

- la réalisation des 20 automotrices en vue de prolonger leur parcours.

- La reconstruction à mi-vie de 75 rames Z 6400 de la banlieue Saint-Lazare commencée en 1999, qui doit se poursuivre jusqu'en 2005. Ensuite, les mêmes travaux seront entrepris sur les Z2N 5600 et Z2N 8800

- La région Centre, désireuse d'aligner le confort des Z 7300 sur le niveau du TER 72500 et TER 73500, fait équiper une automotrice de ce type. Un prototype a été réalisé ; après sa présentation, une série de 15 véhicules a été transformée.

- L'EIMM a pris aussi en compte la maintenance de 22 automotrices Z2 des CFL.

- L'EIMM se trouve aussi confronté aux actes d'incivilité sur le matériel. Pour y remédier, il procède à l'équipement des Z2N en « extrême soirée ", c'est-à-dire la condamnation de deux caisses à partir d'une certaine heure, du pelliculage des peintures intérieures extérieures et au montage de sièges anti-lacération.

- L'EIMM procède aussi aux réparations accidentelles et ponctuelles sur différents matériels.

- Il poursuit son activité sur le parc fret tant en révision qu'en visite ou réparation. Il réalise aussi des travaux de maintenance rémunérés, sur des wagons de particuliers ou de réseaux étrangers.

- Il gère : le service des approvisionnements, un chantier d'examen et nettoyage pour le matériel remorqué à Tours et Orléans, un chantier de réparation sur le triage de Saint-Pierre-des-Corps au lieu-dit "La Vicairerie », un site de visite des wagons sur le triage de SaintPierre-des-Corps, des missions de contrôle dans les industries privées Saint-Amand et Saint-Denis de l'Hotel.

Les automotrices :

Nombre d'automotrices au 31 décembre 2001 : 1023 ; DIF : 697 ; TER : 304 ; Luxembourg : 22.

En permanence 11 rames sont immobilisées en continu dans l'établissement. 109 rames sont sorties de l'EIMM en 2001. 


\begin{tabular}{|l|c|c|c}
\hline Désignation & Nom bre & $\begin{array}{c}\text { Motif } \\
\text { principal }\end{array}$ & Im m obilisation \\
\hline MI2N & 1 & & \\
\hline Z2N & 55 & $\begin{array}{c}\text { Extrême soirée } \\
\text { + climatisation }\end{array}$ & 40 jours \\
\hline Z 5300 & 20 & $\begin{array}{c}\text { Prolongation } \\
\text { parcours }\end{array}$ & 45 jours \\
\hline Z 6400 & 15 & Rénovation & 3 mois \\
\hline
\end{tabular}

\begin{tabular}{|l}
\hline P.R.M. \\
\hline Moteurs : 190 heures/ moteur \\
\hline Bogies : 258,170 heures/ bogie \\
\hline FRET \\
\hline 4741 w agons réparés \\
\hline 245863 w agons visités \\
\hline Approvis ionnement \\
\hline 13300 articles référencés, stockés (consommables, PRMe \\
\hline 155 MF de chiffre d'affaire \\
\hline
\end{tabular}

Source : SNCF

Tableau 3. Nature et répartition de l'activité de l'EIMM de Saint-Pierre-des-Corps en 2001.

\section{L'avenir}

Appelé à devenir atelier de l'ensemble du parc automoteur de la SNCF, l'EIMM de Saint-Pierre-des-Corps prépare son avenir. Actuellement, ses bureaux d'études travaillent en liaison avec les constructeurs pour les plans de maintenance de futur matériel. Selon les prévisions ses ateliers ont 15 années de travail assuré ${ }^{19}$.

\section{Les Établissements Billard}

\section{Les origines : les premières constructions ferroviaires}

C’est en janvier 1920 que M. Pierre Billard fonda la Société BillardChatenay et Cie. À cette époque, l'activité de cet établissement était consacrée à la mécanique générale et plus particulièrement à la fabrication de pièces automobiles. Le développement du moteur à explosion après le conflit de 1914-1918 permettait d'envisager l'avenir avec confiance.

19- Toutes les informations communiquées dans ces pages proviennent de la documentation de l'EIMM. 
L'entreprise s'implanta à Tours, 21, rue du Rempart, dans des locaux non raccordés au chemin de fer.

Les premières productions ferroviaires furent marginales. Elles se bornèrent à la construction de tracteurs de manutention ou de tracteurs de chariots sur les quais de gares. Cette activité attira toutefois l'attention des compagnies ferroviaires qui, dès 1922, lui passèrent commande d'un nombre important de draisines.

\section{La SA des anciens Établissements Billard}

Le développement de l'affaire nécessita l'apport de capitaux nouveaux. Pour y répondre, une société au capital de $5 \mathrm{MF}$ fut créée en 1928. Elle absorba la précédente et prit la dénomination sociale de «SA des anciens Établissements Billard ». Elle se consacra plus particulièrement aux constructions ferroviaires à voies étroites $(0,50,0,60,0,75)$ pour exploitations minières, carrières, etc.

Toutefois, les essais d'adaptation à la voie ferrée des véhicules routiers issus des plans américains amorcés en 1922 par MM. Vernay et Tartary ne laissaient pas M. Billard indifférent. Cela au contraire l'incita à diversifier ses productions ferroviaires. Mais les études et la réalisation de ces nouveaux matériels nécessitèrent aussi de nouveaux ateliers pouvant être raccordés au chemin de fer. C'est ainsi que, profitant de la fermeture du dépôt de l'État à Tours en 1931, l'entreprise s'y installa.

\section{Les nouvelles réalisations}

C'est dans ces locaux mieux adaptés que commencent les nouvelles réalisations. Elles débutent par une série de grosses draisines « coloniales » de $50 \mathrm{cv}$ pour le Congo-Océan. Puis en 1932 commence la construction des séries d'autorails pour les voies ferrées d'intérêt local et chemins de fer coloniaux (fig. 6). Contrairement à MM. Verney et Tartary, l'entreprise construit du matériel neuf. Toutefois, la conception reste très proche de la technique routière. En témoignent ces trois premiers autorails A50 D à deux essieux destinés au réseau départemental de l'Oise. Ils ont l'esthétique d'un autobus avec le capot extérieur non réversible ; par contre, le châssis est de conception purement ferroviaire. La série suivante aura un aspect " plus ferroviaire »; elle bénéficie d'un moteur inclus dans la carrosserie mais la structure reste parallélépipédique hormis les angles arrondis. À partir de 1934, on adopta une structure plus aérodynamique. L'inclinaison des extrémités de caisse est réalisée à partir de 1937.

En 1934, la totalité des ateliers est transférée dans l’ancien dépôt de l'État, rue Robespierre. Seuls sont restés 11, rue du Rempart, les bureaux d'études et administratifs. 
En 1936, une étroite collaboration s'instaura entre les CFD et les Établissements Billard pour l'élaboration de l'autorail A 80 D. La proximité des réseaux CFD nord et sud d'Indre-et-Loire en gare d'Écueillé et le raccordement du réseau sud avec Le Blanc-Argent permettait à l'entreprise d'effectuer les essais de ses constructions en voie métrique (fig. 7). C'est ainsi qu'avant guerre un autorail d'une série destinée à l'exploitation a atteint la vitesse de $115 \mathrm{~km} / \mathrm{h}$ entre Manthelan et La Chapelle-Blanche, sur la ligne Esvres-Ligueil du réseau sud d'Indre-etLoire, battant ainsi un record de vitesse en voie métrique. Il faut aussi noter la construction en 1936-1937 de dix Michelines de 56 places et en 1939 de trois Michelines pour le Mozambique.

Mais les ateliers n'ont toutefois pas complètement abandonné la fabrication de matériel à voie étroite. C'est ainsi qu'en 1939 ils reçurent une commande des services du génie militaire de locomoteurs de 70 à $100 \mathrm{cv}$ pour voie de $0,60 \mathrm{~m}$. Et cette même année, en rompant avec la construction ferroviaire, l'entreprise reçut commande du ministère de l'Air de parties de fuselage de «LEO 45 », le plus gros bombardier du moment.

À cette époque, ils employaient plus de 600 ouvriers. Durant l'Occupation, la société eut une vie industrielle des plus réduites.

En 1944, les ateliers furent touchés par les bombardements alliés. Ce n'est qu'au milieu de l'année 1945 et après de gros efforts que l'entreprise put reprendre une activité normale. Dès lors, elle se consacra à la fabrication de draisines et automotrices à voie étroite tant pour le compte des $\mathrm{CFD}^{20}$ que de la SNCF.

À cette époque de l'après-guerre, l'avenir des petites lignes est encore incertain. Pour diminuer le coût de leurs exploitation, la SNCF essaie d'affermer aux CFD les lignes de Vendée. À cet effet, ils passent commande aux Établissements Billard de cinq autorails type A75D $80 \mathrm{cv}$.

Ces véhicules à deux essieux étaient conçus pour l'exploitation à voie unique. Voyant déjà un risque de privatisation dans cette procédure, les organisations syndicales de cheminots s'opposèrent au projet. Les autorails ne purent rejoindre leur affectation et restèrent à Nantes. Par la suite, ils furent affectés sur d'autres lignes CFD ainsi que sur la ligne Mamers-Saint-Calais.

20- CFD : Chemins de fer départementaux. 


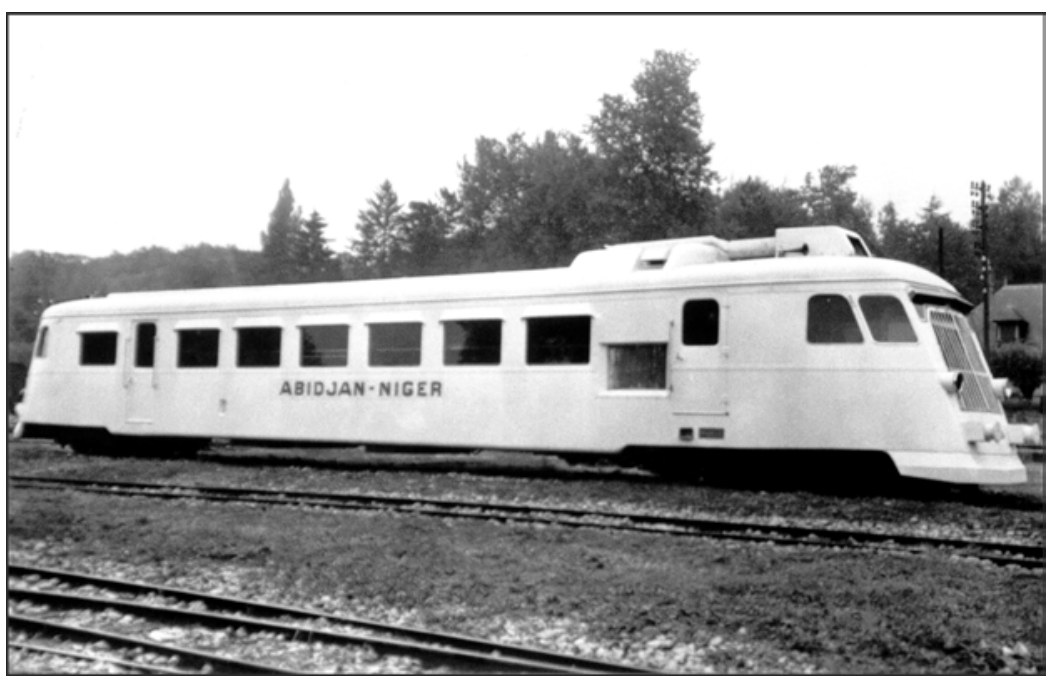

Figure 6. Établissements Billard. Autorail voie de 1 mètre. Réseau BéninNiger. Archives EIMM déposées à la bibliothèque municipale de Saint-Pierre-des-Corps.

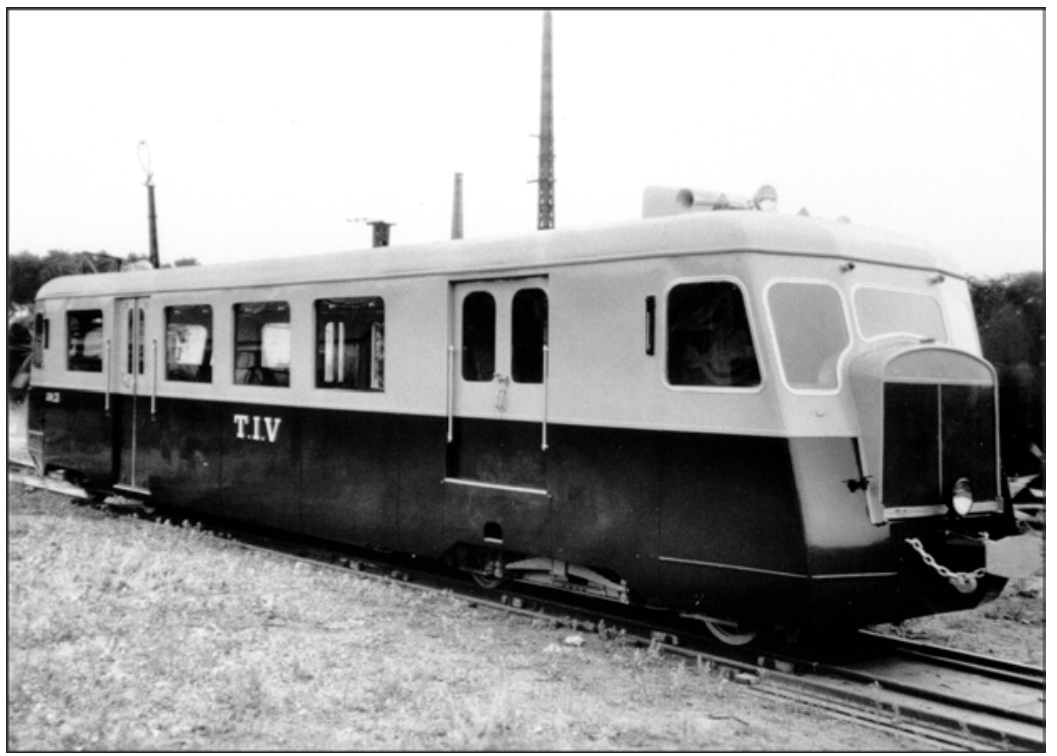

Figure 7. Établissements Billard. Autorail voie de 1 mètre. TIV. Archives EIMM déposées à la bibliothèque municipale de Saint-Pierre-des-Corps. 


\section{L'autorail FNC}

Soucieuse du maintien de l'exploitation par fer des lignes régionales, l'Union nationale des cadres, syndicat affilié à la Fédération nationale des cheminots, présente en 1946 un projet d'autorail léger à deux essieux (fig. 8).

Officiellement désigné FNC, cet autorail était plus confortable que le $\mathrm{A} 75 \mathrm{D}$ (toilettes, compartiment à bagages) et son exploitation maintenait la présence de deux agents. Sa particularité : un poste de conduite unique surélevé sur le côté droit dans le sens de marche. Cette disposition permettait une bonne visibilité dans les deux sens de la marche. Elle allait être conservée sur les séries suivantes (5500-5800 de $150 \mathrm{cv}, 3800-3900$ de $300 \mathrm{cv}$ dit «Picasso »). L'avant-projet a été approuvé par la SNCF qui a confié la réalisation de trois prototypes aux Établissements Billard. Il faut noter la rapidité de la construction du premier prototype :

- diagramme établi le 25 juillet 1946 ;

- congé annuel du 27 au 19 août 1946 ;

- maquette des aménagements intérieurs et du poste conduite présentés le 22 octobre 1946 ;

- début de construction du prototype le 29 août ;

- essais sommaires du 22 au 29 novembre 1946.

Billard n'a construit que les trois prototypes, le reste de la série a été adjugé à la $\mathrm{CGC}^{21}$ et à la $\mathrm{SNAV}^{22}$.

Toutefois, cette pré-série fut suivie d'une commande de trois unités pour le chemin de fer de Merzig à Buschfel. Ces trois derniers autorails étaient accouplables entre eux, et conduits par un seul agent.

\section{La fin de l'entreprise}

Pendant cette même période, l'entreprise a également réalisé une quantité importante de remorques ainsi qu'une partie de la nombreuse lignée des locomotives Y 7100 (fig. 9).

À partir de 1950, les constructions se sont surtout orientées vers l'étranger et les pays francophones. À noter au passage la modernisation de la ligne à crémaillère de Diakolto à Kalavrita dans le Péloponnèse.

21- CGC : Compagnie générale de construction.

22- SNAV : Société nouvelle des ateliers de Vénissieux. 


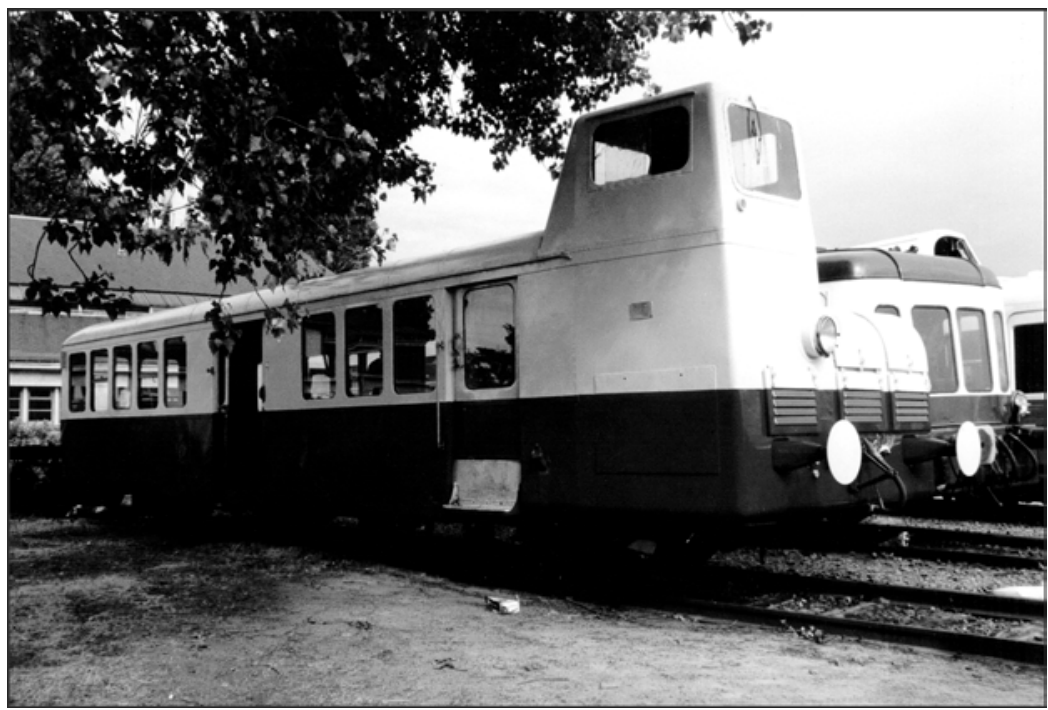

Figure 8. Établissements Billard. Autorail voie normale, type FNC. Cl. B. Monteil.

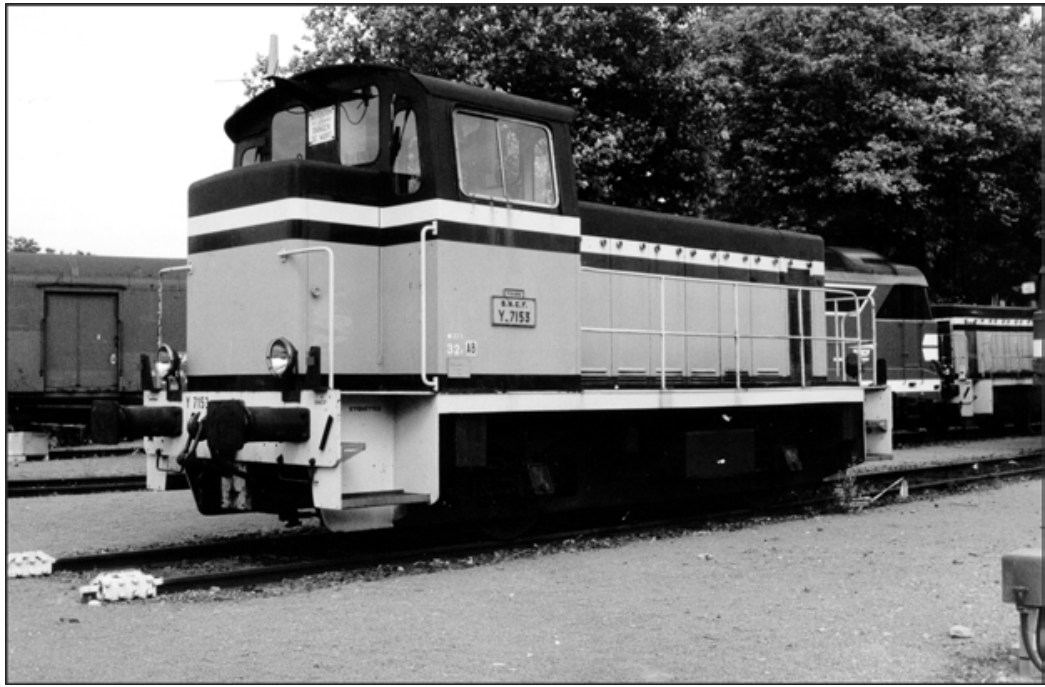

Figure 9. Établissements Billard. Locotrateur SNCF Y.7100. Cl. B. Monteil. 
Comme pour les ateliers voisins précédemment cités, la ville de Tours souhaitait récupérer des terrains afin d'y créer une cité universitaire. La société envisagea alors de poursuivre son activité dans d'anciens bâtiments du PO à Saint-Pierre-des-Corps.

En 1964, alors que le déménagement allait se réaliser, elle se trouva confrontée à des difficultés financières ; tandis qu'une série de matériels pour le chemin de fer franco-Éthiopien était en cours d'achèvement, elle dut cesser son activité le 30 septembre 1946. Elle laissait environ 250 salariés sans emploi.

Les constructions en cours furent reprises par les Établissements Soulé à Bagnère-de-Bigorre pour les voitures et automotrices.

Socofer, fondé en 1966 par d'anciens cadres des Établissements Billard, poursuivit les fabrications. Ils réaliseront en 1983 conjointement avec CFD deux autorails pour la ligne à voie métrique du Blanc-Argent.

Depuis lors, ils se sont spécialisés dans la construction de trains aspirateurs qui équipent les métros de Paris, Milan, Munich, Bâle, NewYork, Séoul. Ils construisent également des trains modulables, des draisines, des véhicules rail-route pour la maintenance des infrastructures. Le travail de cet établissement consiste en la réhabilitation de matériel (500 bogies pour la RATP, MF 67 et 77,115 kits moteurs pour autorails X 2100 et 2200).

Actuellement, l'entreprise emploie sur les sites de Tours et Jouélès-Tours environ 100 personnes.

\section{Sources}

\section{Les ateliers de Tours}

- Maurice Gaucher, "L'avenir du chemin de fer de Tours », art. imprimé, coll. privée.

- Maurice Maillet, Les "Pacifics" du PO-Midi et l'œurre d'André Chapelon, Breil-sur-Roya, Ed. du Cabri, 1980.

- Bernard Collardey ; André Rasserie, Les Locomotives à vapeur unifiées 241 P, 240 P, 150, Paris, Ed. La vie du rail et des Transports, 2001, $199 \mathrm{p}$.

\section{Les ateliers de Saint-Pierre-des-Corps}

- Histoire de l'Atelier de réparation du Matériel Ferroviaire de St-Pierredes-Corps, section syndicale CGT des cheminots de l'EIMM de Saint-Pierre-des-Corps. 


\section{Les ateliers de Vitry}

- Pascal Grassart, «Vitry, un atelier sur la selette », La Vie du Rail, $\mathrm{n}^{\circ} 2466$ (19-25 octobre 1994), p. 6-7.

\section{Les Établissements Billard}

- Revue MTVS, n² 24-4, 1982.

- Archives Billard, détenues par Socofer,Tours. 